\title{
GROUPE DE HEISENBERG ET RÉALITÉ
}

\author{
P. DELIGNE
}

1. Soit $H$ le groupe de Heisenberg complexe, extension de $\mathbb{C}^{2}$ par $\mathbb{C}^{*}$. Il admet des coordonnées $(x, y, \lambda)$, la loi de groupe étant donnée par

$$
(x, y, \lambda)\left(x^{\prime}, y^{\prime}, \lambda^{\prime}\right)=\left(x+x^{\prime}, y+y^{\prime}, \lambda \lambda^{\prime} \exp \left(\pi i\left(x y^{\prime}-x^{\prime} y\right)\right)\right) \text {. }
$$

L'involution

$$
\tau:(x, y, \lambda) \mapsto(-x,-y, \lambda) .
$$

est un automorphisme de $H$.

Le système de coordonnées $x, y, \lambda: H \rightarrow \mathbb{C}^{2} \times \mathbb{C}^{*}$ met en évidence la section $\mathbb{C}^{2} \times\{1\}$ de la projection de $H$ sur $\mathbb{C}^{2}$. Sur cette section $\mathbb{C}^{2} \times\{1\}, \tau(g)=g^{-1}$. Chaque droite $D \subset \mathbb{C}^{2}$ a pour relèvement par cette section un sous-groupe à un paramètre de $H$, qu'on notera encore $D$. Le commutateur $(u, v)=u v u^{-1} v^{-1}$ de deux éléments $u=\left(u_{1}, u_{2}\right)$ et $v=\left(v_{1}, v_{2}\right)$ de $\mathbb{C}^{2}$ est $\exp (2 \pi i\langle u, v\rangle) \in \mathbb{C}^{*}$ avec

$$
\langle u, v\rangle=u_{1} v_{2}-u_{2} v_{1}
$$

Soit $H_{\mathbb{R}}$ la forme réelle: $x, y \in \mathbb{R},|\lambda|=1$. Le groupe $\operatorname{SL}(2, \mathbb{C})$ agit sur $H$ et $\operatorname{SL}(2, \mathbb{R})$ agit sur $H_{\mathbb{R}}$, identifié ensemblistement à $\mathbb{R}^{2} \times U^{1}$.

On sait (théorème de Stone-von Neumann [2]) que $H_{\mathbb{R}}$ admet une unique représentation unitaire irréductible $V$ pour laquelle $\lambda \in U^{1}$ agit par multiplication par $\lambda$. Dans le modèle de Schrödinger de $V$, on a $V=L^{2}(\mathbb{R})$ et l'action de $H_{\mathbb{R}}$ est:

$$
\begin{aligned}
& (x, 0,1): f(z) \mapsto f(z+x) \\
& (0, y, 1): f(z) \mapsto e^{2 \pi i y z} f(z) .
\end{aligned}
$$

De l'unicité de $V$ on déduit une action projective unitaire de $\operatorname{SL}(2, \mathbb{R})$ sur $V$, compatible à l'action de $\operatorname{SL}(2, \mathbb{R})$ sur $H_{\mathbb{R}}$ (A. Weil [3], D. Shale [1]).

Pour l'action de $H_{\mathbb{R}}$ sur $V \simeq L^{2}(\mathbb{R})$, les vecteurs $C^{\infty}$ i.e. les $v \in V$ tels que $h \mapsto h v$ soit $C^{\infty}$ en $h \in H_{\mathbb{R}}$, forment l'espace de Schwartz $\mathscr{S}$ des fonctions $C^{\infty}$ à décroissance rapide sur $\mathbb{R}$. Les vecteurs holomorphes, i.e. les $v \in V$ tels que $h \mapsto h v$ se prolonge en une application holomorphe de $H$ dans $V$,

Received by the editors August 3, 1990.

1980 Mathematics Subject Classification (1985 Revision). Primary 22E45. 
sont les fonctions $L^{2}$ sur $\mathbb{R}$ se prolongeant en une fonction holomorphe sur $\mathbb{C}$ telle que, dans toute bande horizontale $|\operatorname{Im} z|<A_{1}$ on ait pour tout $A_{2} \in \mathbb{R}$

$$
|f(z)| \leq 0\left(\exp \left(-A_{2}|z|\right)\right) \text {. }
$$

Le groupe complexe $H$ agit sur l'espace $V^{\text {hol }}$ des vecteurs holomorphes par les formules (1.4).

On ne dispose pas pour l'action holomorphe de $H$ sur $V^{\text {hol }}$ de la même unicité que pour l'action unitaire de $H_{\mathbb{R}}$ sur $V$. Sinon, l'action projective de $\operatorname{SL}(2, \mathbb{R})$ sur $V$ se prolongerait en une action projective de $\operatorname{SL}(2, \mathbb{C})$ sur $V^{\text {hol }}$, cette action se relèverait en une vraie action de $\operatorname{SL}(2, \mathbb{C})$, car $\operatorname{SL}(2, \mathbb{C})$ est simplement connexe, et l'action projective de $\operatorname{SL}(2, \mathbb{R})$ se relèverait en une vraie action. Ce n'est pas le cas: seul le revêtement double de $\operatorname{SL}(2, \mathbb{R})$ agit.

Notre but est d'expliciter ce qui se passe.

2. Soit $S=\mathbb{P}^{1}(\mathbb{C})$ l'espace des droites de $\mathbb{C}^{2}$. Pour $D \in S$, relevé dans $H$ comme au $n^{0} 1$, soit $W_{D}$ l'espace des fonctions holomorphes sur $H$ vérifiant

$$
\varphi(\lambda d h)=\lambda \varphi(h)
$$

pour $\lambda \in \mathbb{C}^{*}, d \in D$, muni de l'action de $H: g: \varphi(h) \mapsto \varphi(h g)$. Pour $E \neq D$, la restriction à $E$ est une bijection

$$
r_{E}: W_{D} \stackrel{\sim}{\rightarrow} \mathscr{O}(E),
$$

$\mathscr{O}(E)$ étant l'espace des fonctions holomorphes sur $E$.

Si $R$ est une représentation holomorphe de $H$, sur laquelle chaque $\lambda \in \mathbb{C}^{*}$ agit par $\lambda$, et $\omega$ une forme linéaire $D$-invariante sur $R$,

$$
w \mapsto \omega(h w)
$$

est un morphisme de $R$ dans $W_{D}$.

3. Exemple. Soient comme aux $n^{0} 1,2 \quad V$ la représentation unitaire irréductible de $H_{\mathbb{R}}, \mathscr{S} \subset V$ l'espace de ses vecteurs $C^{\infty}$ et soit $\mathscr{S}^{\prime} \supset V$ le dual de l'espace des vecteurs $C^{\infty}$ de la représentation duale (dans le modèle de Schrödinger: l'espace des distributions tempérées).

Soit $D_{t} \in S$ la droite engendrée par $(-t, 1)$. Si $\operatorname{Im} t>0$, la forme linéaire, écrite dans le modèle de Schrödinger,

$$
\omega_{t}: f \mapsto \frac{1}{\sqrt{t / i}} \int_{\mathbb{R}} f(x) \exp \left(-\pi i x^{2} / t\right) d x
$$

est définie sur $V$, et même sur $\mathscr{S}^{\prime}$. La forme $\omega_{t}$ est un vecteur holomorphe du dual de $V$, fixe par $D_{t}$. En terme du générateur infinitésimal $-t \partial_{x}+2 \pi i x$ de $D_{t}$, l'invariance s'écrit

$$
\begin{aligned}
\int_{\mathbb{R}}\left[\left(-t \partial_{x}+2 \pi i x\right) f(x)\right] \cdot \exp \left(-\pi i x^{2} / t\right) d x \\
\quad=\int_{\mathbb{R}} f(x) \cdot\left(t \partial_{x}+2 \pi i x\right) \exp \left(-\pi i x^{2} / t\right) d x=0 .
\end{aligned}
$$


$\operatorname{Par}(2.2), \omega_{t}$ définit

$$
\Omega_{t}: V^{\mathrm{hol}} \rightarrow W_{D_{t}}
$$

qui se prolonge à $V$ et même à $\mathscr{S}^{\prime}$. Soit $E$ l'axe des $x$. L'application $r_{E} \Omega_{t}: V \rightarrow \mathscr{O}(E)$ envoie $f \in V$ sur

$$
f(x, t)=r_{E} \Omega_{t}(f) \quad(\operatorname{Im} t>0)
$$

solution de l'équation de la chaleur

$$
\left(\partial_{t}-\frac{1}{4 \pi i} \partial_{x}^{2}\right) f(x, t)=0 .
$$

Pour $t$ réel, la forme $\omega_{t}$ reste définie sur $\mathscr{S} \subset V$. Pour $t=0$, c'est $f \mapsto f(0)$. Pour $t=\infty$, c'est $\int f(x) d x$. L'homomorphime $\Omega_{t}$ reste défini sur $V^{\text {hol }}$. Pour $t=0, r_{E} \Omega_{0}$ est, dans le modèle de Schrödinger, l'identité.

Supposons $\operatorname{Im}(t)>0$. Soit $\bar{D}_{t}$ la complexe conjuguée de $D_{t}$. Dans la droite affine $S-\left\{\bar{D}_{t}\right\}$, le "demi-plan" $\left\{D_{u} \mid \operatorname{Im} u>0\right\}$ est un disque de centre $D_{t}$. L'application $r_{D_{t}} \circ \Omega_{t}: V \rightarrow W_{D_{t}} \rightarrow \mathscr{O}\left(\bar{D}_{t}\right)$ fournit le modèle holomorphe de $V:$ l'action de $H$ est donnée par

$$
\begin{aligned}
& d \in \bar{D}_{t}: f(x) \mapsto f(x+d) \\
& e \in D_{t}: f(x) \mapsto \exp (-2 \pi i\langle e, x\rangle) f(x),
\end{aligned}
$$

la structure hilbertienne où $\langle f, f\rangle$ est l'intégrale de

$$
f(d) f(d)^{-} \exp (2 \pi i\langle d, \bar{d}\rangle)
$$

est invariante par $H_{\mathbb{R}}$ et $r_{D_{t}} \circ \Omega_{t}$ identifie $V$ à l'espace des $f$ de norme finie. Pour $d=(-\bar{t} z, z)$, le facteur $\exp (2 \pi i\langle d, \bar{d}\rangle)=$ commutateur de $d$ et $\bar{d}$ est

$$
\exp (2 \pi i\langle d, \bar{d}\rangle)=\exp \left(2 \pi i(t-\bar{t})|z|^{2}\right) .
$$

4. Fixons $E$. Les isomorphimes $r_{E}(2.1)$ permettent de considérer les $W_{D}$ $(D \neq E)$ comme formant une famille de représentations de $H$ opérant dans l'espace fixe $\mathscr{O}(E)$, l'action de $H$ dépendant holomorphiquement de $D$. Explicitons la dépendance en $E$ de $r_{E}$. Prenons des coordonnées comme au $n^{0} 1$ où $E$ soit l'axe des $x$. Soient $E^{\prime}$ engendré par $(1, u), D$ engendré par $(-t, 1)$. On prend $x$ comme coordonnée sur $E$ et $E^{\prime}$. On a

$$
\left(x^{\prime}, u x^{\prime}, 1\right)=\left(-t u x^{\prime}, u x^{\prime}, 1\right)(x, 0,1)(0,0, \lambda)
$$

pour $(1+t u) x^{\prime}=x$ et $\lambda=\exp \left(\pi i u x x^{\prime}\right)$, de sorte que $r_{E^{\prime}} r_{E}^{-1}$ envoye $f_{E} \in \mathscr{O}(E)$ $\operatorname{sur} f_{E^{\prime}} \in \mathscr{O}\left(E^{\prime}\right)$ avec $f_{E^{\prime}}\left(x^{\prime}\right)=\lambda f_{E}(x)$ :

$$
f_{E^{\prime}}\left(\frac{x}{1+t u}\right)=\exp \left(\frac{\pi i u}{1+t u} x^{2}\right) f_{E}(x) \text {. }
$$

Cette formule est holomorphe en $t$ : les $W_{D}$ forment un fibré holomorphe $\mathscr{W}$ (de dimension infinie) sur $S$, sur lequel $H$ agit. 
Pour comprendre (4.1), on peut noter que le point de $E$ de coordonnée $x$ a même image dans $\mathbb{C}^{2} / D$ que le point de $E^{\prime}$ de coordonnée $x / 1+t u$.

De (4.1) on déduit que la condition de croissance suivante sur $v \in W_{D}$ est indépendante des choix de $E \neq D$ et de la coordonnée $z$ sur $E$

$$
\text { pour } f=r_{E}(v), \exists A|f(z)| \leq 0\left(\exp \left(A|z|^{2}\right) .\right.
$$

Soit $W_{D}^{0} \subset W_{D}$ défini par cette condition. Les $W_{D}^{0}$ forment un sous-fibré holomorphe $\mathscr{W}^{0}$ en représentations holomorphes de $H$ du fibré $\mathscr{W}$.

5. Proposition. Soit $T>0$ et considérons les conditions suivantes sur une fonction entière $f$ :

(i) $T$ Il existe une fonction holomorphe $g(z, t)(z \in \mathbb{C},|t|<T)$ vérifiant l'équation de la chaleur $\left(\partial_{t}-\partial_{z}^{2}\right) g=0$, avec la condition initiale $g(z, 0)=$ $f(z)$;

(ii) $_{T}|f(z)| \leq 0\left(\exp \left(|z|^{2} / 4 T\right)\right)$;

(iii) $_{T} \int f(z) f(\bar{z}) \exp \left(-|z|^{2} / 2 T\right)|d z \wedge d \bar{z}|<\infty$.

Alors, si $T<T_{1}$, chacune de $(\mathrm{i})_{T_{1}}$, $\left({ }^{\mathrm{ii}}\right)_{T_{1}}$, $\left({ }^{\mathrm{iii}}\right)_{T_{1}}$ implique chacune de $(\mathrm{i})_{T}$, (ii) $_{T}$, (iii) $)_{T}$.

Preuve. $(\mathrm{i})_{T_{1}} \Rightarrow(\mathrm{ii})_{T}$. L'équation de la chaleur reste vérifiée par les dérivées de $g$ et on a donc

$$
\begin{aligned}
\partial_{z}^{2 n} g & =\partial_{t}^{n} g, \\
\partial_{z}^{2 n+1} g & =\partial_{t}^{n}\left(\partial_{z} g\right) .
\end{aligned}
$$

Puisque $g(0, t)$ est holomorphe pour $|t|<T_{1}$, si $T<T_{2}<T_{1}$, on a en $(0,0)$

$$
\begin{aligned}
\left|\partial_{t}^{n} g / n !\right| & \leq 0\left(1 / T_{2}^{n}\right), \text { d'où } \\
\left|\partial_{z}^{2 n} g\right| & \leq 0\left(n ! / T_{2}^{n}\right)
\end{aligned}
$$

et une estimation analogue pour $\partial_{z}^{2 n+1} g$. La série de Taylor de $f$ donne alors

$$
|f(z)| \leq 0\left(\sum \frac{n !}{(2 n) !} \frac{1}{T_{2}^{n}}|z|^{2 n}\right) .
$$

On a

$$
\frac{1}{2 n+1} 2^{2 n} \leq \frac{(2 n) !}{(n !)^{2}} \leq 2^{2 n}
$$

d'où

$$
|f(z)| \leq 0\left(\sum 2^{-2 n} \frac{1}{T^{n}} \frac{|z|^{2 n}}{n !}\right)
$$

et cette somme est $\exp \left(|z|^{2} / 4 T\right)$.

${ }^{(i i)} T_{T_{1}} \Rightarrow(\mathrm{i})_{T}$. Il suffit de prendre $g$ donné par le noyau de l'équation de la chaleur:

$$
g(z, t)=\int_{\sqrt{t} \mathbb{R}} f(z+x) \cdot \frac{1}{2 \sqrt{\pi t}} \exp \left(-x^{2} / 4 t\right) d x .
$$


Changer $\sqrt{t}$ en $-\sqrt{t}$ change l'orientation du cycle $\sqrt{t} \mathbb{R}$ et le signe de l'intégrand, de sorte que l'intégrale ne change pas. La condition (ii) assure la convergence pour $|t|<T$.

La relation entre (ii) et (iii) est claire: (ii) $T_{1} \Rightarrow\left(\right.$ iii) $T_{T}$ et que (iii) $T_{1} \Rightarrow\left(\right.$ ii) ${ }_{T}$ se vérifie en écrivant $f(z)$ comme moyenne de ses valeurs sur un disque de centre $z$ et de rayon $1 /|z|$, et en évaluant cette moyenne par Cauchy-Schwartz.

6. En hommage au théorème de Stone-von Neumann, le fibré $\mathscr{W}$ sur $S$ est, projectivement, muni d'une connexion holomorphe pour laquelle l'action de $H$ est horizontale.

Précisons: "projectivement." Sur la droite projective $S$, on dispose du faisceau inversible ample $\mathscr{O}(1)$. Soit $\mathscr{O}(-1)$ son dual. Une racine carrée $\mathscr{O}\left(-\frac{1}{2}\right)$ de $\mathscr{O}(-1)$ est un faisceau inversible $L$ muni d'un isomorphisme $L^{\otimes 2} \stackrel{\sim}{\rightarrow} \mathscr{O}(-1)$. Localement, une telle racine carrée existe et, localement, deux racines carrées sont isomorphes, mais l'isomorphisme n'est pas unique: ambiguïté \pm 1 . Cette ambiguïté obstrue l'existence globale de $\mathscr{O}\left(-\frac{1}{2}\right)$. Ce n'est pas sur $\mathscr{W}$, mais sur $\mathscr{W}\left(-\frac{1}{2}\right):=\mathscr{W} \otimes \mathscr{O}\left(-\frac{1}{2}\right)$ qu'on a une connexion:

$$
\nabla: \mathscr{W}\left(-\frac{1}{2}\right) \rightarrow \mathscr{W}\left(-\frac{1}{2}\right) \otimes \Omega^{1}
$$

Noter que si $\mathscr{O}\left(-\frac{1}{2}\right)^{\prime}$ et $\mathscr{O}\left(-\frac{1}{2}\right)^{\prime \prime}$ sont deux racines carrées de $\mathscr{O}(-1)$, et $\nabla$ une connexion sur $\mathscr{W} \otimes \mathscr{O}\left(-\frac{1}{2}\right)^{\prime}$, son transporté sur $\mathscr{W} \otimes \mathscr{O}\left(-\frac{1}{2}\right)^{\prime \prime}$ par $\alpha: \mathscr{O}\left(-\frac{1}{2}\right)^{\prime} \stackrel{\sim}{\rightarrow} \mathscr{O}\left(-\frac{1}{2}\right)^{\prime \prime}$ (un isomorphisme de racines carrées de $\mathscr{O}(-1)$ ) ne dépend pas du choix de $\alpha$, qu'on ne peut changer localement que par une constante \pm 1 . La notion de "connexion sur $\mathscr{W}\left(-\frac{1}{2}\right)$ " est donc bien définie, indépendamment de l'existence globale ou du choix de $\mathscr{O}\left(-\frac{1}{2}\right)$.

Soit $D \in S$ et $t$ un vecteur tangent en $D$. Pour $s$ une section locale de $\mathscr{W}\left(-\frac{1}{2}\right)$, il s'agit de définir $\nabla_{t} s$ dans la fibre de $\mathscr{W}\left(-\frac{1}{2}\right)$ en $D$. On procédera comme suit: après avoir fait un choix auxiliaire $u$, on définira $\nabla_{u} s$ pour $s$ une section locale de $\mathscr{W}$, ou de $\mathscr{O}\left(-\frac{1}{2}\right)$. Cette dérivation $\nabla_{u}$ dépend de $u$, avec une dépendance en $u$ de la forme

$$
\nabla_{u^{\prime}}(s)=\nabla_{u}(s)+a s(D),
$$

$a$ prenant des valeurs opposées pour $\mathscr{W}$ et pour $\mathscr{O}\left(-\frac{1}{2}\right):$ pour $s=s_{1} s_{2}, s_{1}$ section locale de $\mathscr{W}$ et $s_{2}$ de $\mathscr{O}\left(-\frac{1}{2}\right)$,

$$
\nabla_{t}(s):=\nabla_{u}\left(s_{1}\right) s_{2}(D)+s_{1}(D) \nabla_{u}\left(s_{2}\right)
$$

est indépendant du choix de $u$.

Soit $D \in S: D$ est une droite de $\mathbb{C}^{2}$. Un vecteur tangent $t$ en $D \in S$ s'identifie à une application linéaire $t: D \rightarrow \mathbb{C}^{2} / D$, i.e. à un élément de $\left(\mathbb{C}^{2} / D\right)^{\otimes 2}:$ à $u^{\prime} \otimes v^{\prime} \in\left(\mathbb{C}^{2} / D\right)^{\otimes 2}$ attacher $t(d)=\langle u, d\rangle v^{\prime}$ pour $u$ un quelconque représentant de $u^{\prime}$. Notre donnée auxiliaire sera celle de $\tilde{t}: D \rightarrow \mathbb{C}^{2}$ relevant $t$, i.e. d'un relèvement de $t$ à $\mathbb{C}^{2} / D \otimes \mathbb{C}^{2}$. Nous l'écrirons $\tilde{t}=u^{\prime} \otimes u$, avec $u \in \mathbb{C}^{2}$ d'image $u^{\prime}$ dans $\mathbb{C}^{2} / D$. 
L'espace tangent à l'origine de $H$ est $\mathbb{C}^{3}=\mathbb{C}^{2} \times \mathbb{C}$. Pour $z$ dans cet espace tangent, soit $\delta_{z}$ le champ de vecteurs invariant à droite correspondant. On a

$$
\left[\delta_{x}, \delta_{y}\right]=-\delta_{[x, y]},
$$

où figure à droite le crochet dans l'algèbre de Lie de $H$. Pour $x, y \in \mathbb{C}^{2}$, calculant $[x, y]$ comme un commutateur infinitésimal, on en déduit que

$$
\left[\delta_{x}, \delta_{y}\right]=-2 \pi i\langle x, y\rangle \delta_{(0,0,1)} .
$$

Soit $\mathscr{U}_{1}$ le quotient de l'algèbre enveloppante de l'algèbre de Lie des champs de vecteurs invariants à droite par la relation $\delta_{(0,0,1)}=1$. Dans $\mathscr{U}_{1}$, on déduit de (6.2) que, pour $d \in D$,

$$
\left[\frac{-\delta_{u}^{2}}{4 \pi i}, \delta_{d}\right]=\frac{1}{4 \pi i} \cdot 2 \cdot 2 \pi i\langle u, d\rangle \delta_{u}=\langle u, d\rangle \delta_{u}=\delta_{i(d)} .
$$

Calculons au premier ordre autour de $D$. Pour $\varepsilon^{2}=0$, et $\varphi(g)$ dans $W_{D}$ : $\delta_{d} \varphi=0$, on a

$$
\delta_{d+\varepsilon \tilde{l}(d)}\left[\varphi(g)+\varepsilon \frac{-\delta_{u}^{2}}{4 \pi i} \varphi(g)\right]=\varepsilon\left[\delta_{\tilde{t}(d)} \varphi(g)+\delta_{d} \frac{-\delta_{u}^{2}}{4 \pi i}\right] \varphi(g)=0
$$

car $\delta_{d}\left(-\delta_{u}^{2} / 4 \pi i\right) \varphi=-\left[-\delta_{u}^{2} / 4 \pi i, \delta_{d}\right] \varphi$ : appliquer (6.3). Au premier ordre autour de $D$, la fonction de $g$ et $D^{\prime}$ définie par $\varphi(g, D+\varepsilon t)=\varphi(g)+$ $\varepsilon\left(-\delta_{u}^{2} / 4 \pi i\right) \varphi(g)$ est donc une section du fibré $\mathscr{W}$. Si $\varphi\left(g, D^{\prime}\right)$ est une section locale de $\mathscr{W}$, ceci permet de définir la dérivée $\nabla_{u} \varphi$ dans $\mathscr{W}_{D}$ par

$$
\nabla_{u} \varphi=\delta_{t} \varphi\left(g, D^{\prime}\right)+\left(\delta_{u}^{2} / 4 \pi i\right) \varphi(g, D) .
$$

Calculons la dépendance de (6.5) en $u$. Si $u$ est remplacé par $u+x \quad(x \in D)$, on a dans $\mathscr{U}_{1}$

$$
\begin{aligned}
\delta_{u+x}^{2} & =\left(\delta_{u}+\delta_{x}\right)^{2}=\delta_{u}^{2}+2 \delta_{u} \delta_{x}+\left[\delta_{x}, \delta_{u}\right] \\
& =\delta_{u}^{2}+2 \delta_{u} \delta_{x}+2 \pi i\langle u, x\rangle .
\end{aligned}
$$

Pour $\varphi \in V_{D}$, on a $\delta_{x} \varphi=0$ et (6.6) se simplifie en

$$
\delta_{u+x}^{2} \varphi=\delta_{u}^{2} \varphi+2 \pi i\langle u, x\rangle \varphi,
$$

d'où

$$
1 / 4 \pi i \delta_{u+x}^{2} \varphi=1 / 4 \pi i \delta_{u}^{2} \varphi+\frac{1}{2}\langle u, x\rangle \varphi .
$$

Le choix de $u$ permet aussi de définir la dérivée $\nabla_{u}$ par rapport à $t$ d'une section locale de $\mathscr{O}(1)$. Une section de $\mathscr{O}(1)$ sur $U \subset S$ est une fonction homogène de degré 1 sur l'image inverse de $U$ dans $\mathbb{C}^{2}$, et on dérive par rapport à $\langle u, d\rangle u$ pour obtenir une fonction homogène de degré 1 sur $D$. Quand on change $u$ en $u+x$,

$$
\begin{aligned}
\nabla_{u+x}(s) & =\langle u+x, d\rangle \delta_{u+x} s \\
& =\nabla_{u}(s)+\langle u, x\rangle s .
\end{aligned}
$$


Cette dérivation en induit une sur $\mathscr{O}(-1 / 2)$, avec

$$
\nabla_{u}\left(s^{-1 / 2}\right)=-1 / 2 \nabla_{u}(s) \cdot s^{-3 / 2}
$$

et pour dépendance en $u, s$ étant cette fois une section locale de $\mathscr{O}\left(-\frac{1}{2}\right)$

$$
\nabla_{u+x}(s)=\nabla_{u}(s)-\frac{1}{2}\langle u, x\rangle s .
$$

Les dépendances en $u$ de (6.8) et (6.9) se neutralisent pour fournir une dérivation $\nabla_{t}$ pour $\mathscr{W}\left(-\frac{1}{2}\right)$.

La connexion $\nabla$ étant définie en terme de champs de vecteurs invariants à droite sur $H$, elle commute à l'action de $H$.

7. Explicitons la connexion du $n^{\circ} 6$ en coordonnées. Choisissons des coordonnées comme au $n^{\circ} 1$ et soit $E$ l'axe des $x$. Sur $S, \mathscr{O}(1)$ admet une section $s$ ayant un zéro simple en $E$. Elle est unique à un facteur près et trivialise $\mathscr{O}(1)$ sur $S-E$. Il existe donc une racine carrée $\mathscr{O}\left(-\frac{1}{2}\right)$ sur $S-E$, trivialisée par une racine carrée $s^{-1 / 2}$ de $s^{-1}$. Par une telle trivialisation, la connexion cherchée se transporte en une connexion sur le fibré $\mathscr{W}$. Nous allons la calculer.

Soit $D_{t}$ la droite engendrée par $(-t, 1)$. Par $r_{E}(1.2)$, une section locale de $\mathscr{W}$ s'identifie à une fonction $f(z, t)$ holomorphe en $z$ et $t$. Calculons sa dérivée par rapport à $t$. Dans le $n^{\circ} 6$, on peut prendre pour

$$
\tilde{t}: D_{t} \rightarrow \mathbb{C}^{2}:(-t z, z) \mapsto(-1,0) z \text {; this is } d \mapsto-\langle(1,0), d\rangle \cdot(1,0) \text {. }
$$

La dérivation correspondante de $\mathscr{O}(1)$ annule $s$, de sorte qu'on a simplement

$$
\nabla_{t} f=\left(\partial_{t}-\partial_{z}^{2} / 4 \pi i\right) f
$$

et les sections locales horizontales sont les solutions de l'équation de la chaleur

$$
\left(\partial_{t}-\partial_{z}^{2} / 4 \pi i\right) f=0 .
$$

$\mathrm{Du} n^{\circ} 5$, on déduit que par $s \in W_{D}^{0}\left(-\frac{1}{2}\right)$ il passe une section locale horizontale de $\mathscr{W}\left(-\frac{1}{2}\right)$. C'est automatiquement une section de $\mathscr{W}^{0}\left(-\frac{1}{2}\right)$. Elle existe dans un voisinage de $D$ d'autant plus grand que $s$ est à croissance plus lente. Noter qu'en dimension infinie une connexion ne définit pas nécessairement une trivialisation locale, même si par chaque point passe une section horizontale locale.

La connexion $\nabla$ sur $\mathscr{W}^{0}\left(-\frac{1}{2}\right)$ en définit une sur le fibré en espaces projectifs correspondant $\mathbb{P}\left(\mathscr{W}^{0}\right)=\mathbb{P}\left(\mathscr{W}^{0}\left(-\frac{1}{2}\right)\right)$. Les sections locales horizontales sont les images des sections horizontales non nulles de $\mathscr{W}^{0}\left(-\frac{1}{2}\right)$. Bien que la sphère $S$ soit simplement connexe, on a:

8. Surprise. Le fibré en espaces projectifs $\mathbb{P}\left(\mathscr{W}^{0}\right)$ sur $S$ n'a aucune section horizontale globale.

L'argument du $n^{0} 1$, permettrait de le prévoir: l'appliquer à la représentation projective de $\operatorname{SL}(2, \mathbb{C})$ donnée par l'action de $\operatorname{SL}(2, \mathbb{C})$ sur l'espace projectif des sections horizontales globales de $\mathbb{P}\left(\mathscr{W}^{0}\right)$. 
$1^{\text {ère }}$ preuve. Divisons $S$ en deux hémisphères $S_{1}$ et $S_{2}$ et choisissons $\mathscr{O}\left(-\frac{1}{2}\right)_{i}$ sur $S_{i}$. Sur $S_{1} \cap S_{2}$, on dispose localement de $\alpha: \mathscr{O}\left(-\frac{1}{2}\right)_{1} \stackrel{\sim}{\longrightarrow} \mathscr{O}\left(\frac{1}{2}\right)_{2}$, défini au signe près, mais $\alpha$ ne peut être défini globalement que sur le revêtement double du cercle $S_{1} \cap S_{2}$ : monodromie -1 . S'il existait des sections projectives globales horizontales de $\mathbb{P}\left(V^{\circ}\right)$, il existerait des sections horizontales $f_{1}, f_{2}$ de $\mathscr{W}^{0}\left(-\frac{1}{2}\right)$ sur $S_{1}$ et $S_{2}$ qui, sur $S_{1} \cap S_{2}$ coïncideraient au signe près. Sur $S_{1} \cap S_{2}$, ceci permettrait de normaliser $\alpha: \mathscr{O}\left(\frac{1}{2}\right)_{1} \stackrel{\sim}{\longrightarrow} \mathscr{O}\left(-\frac{1}{2}\right)_{2}$ par $\alpha\left(f_{1}\right)=f_{2}$, contredisant l'inexistence de $\alpha$ sur $S_{1} \cap S_{2}$.

$2^{\text {ème }}$ preuve. Choisissons des coordonnées comme au $n^{\circ} 1$. Soit $E$ l'axe des $x$ et $D$ celui des $y$. Comme en $n^{\circ} 2$, identifions $W_{D}$ à l'espace des fonctions entières $f(z)$. Soit $f \neq 0$ dans $W_{D}$. Par la proposition, pour qu'il existe sur $S-E$ une section horizontale de $P\left(\mathscr{W}^{0}\right)$ passant par $f$, il faut et il suffit que $f$ vérife la condition de croissance

$$
\forall \varepsilon>0|f(z)| \leq 0\left(\exp \left(\varepsilon|z|^{2}\right)\right)
$$

Si $E^{\prime}$ est une autre droite, d'après (4.1), l'existence d'une section horizontale sur $S-E^{\prime}$ passant par $f$ se traduit par une condition de croissance

$$
\forall \varepsilon>0\left|f(z) \exp \left(a z^{2}\right)\right| \leq 0\left(\exp \left(\varepsilon|z|^{2}\right)\right.
$$

avec $a \neq 0$ convenable.

Posons $g(z)=f(z) \exp \left(a z^{2} / 2\right)$. Par (8.1) et (8.2), $|g(z)|$ est dominé par un multiple tant de $\left|\exp \left(\varepsilon|z|^{2}+a z^{2} / 2\right)\right|$ que de $\left|\exp \left(\varepsilon|z|^{2}-a z^{2} / 2\right)\right|$ de sorte que dans toutes les directions, sauf celles pour lesquelles $a z^{2}$ est purement imaginaire, $g$ est à décroissance exponentielle quadratique. Dans toutes les directions, on a uniformément une croissance au plus exponentielle quadratique. Par Phragmen-Lindelöf, $g$ est à décroissance exponentielle quadratique dans toutes les directions, donc est nulle, et $f=0$.

9. Dans les coordonnées du $n^{\circ} 1$, soit $D_{t}$ la droite engendrée par $(-t, 1)$. Soit $d=(-t z, z)$. On a pour le commutateur de $d$ et $\bar{d}$

$$
(d, \bar{d})=\exp \left(-2 \pi i(t-\bar{t})|z|^{2}\right) \in \mathbb{C}^{*} \quad:
$$

le commutateur est réel positif et

$$
(d, \bar{d})>1 \Longleftrightarrow \operatorname{Im}(t)>0
$$

Les droites $D$ avec pour $d$ engendrant $D(d, \bar{d})>1$ forment donc un disque dans $S$, dit attaché à la structure réelle $H_{R}$.

Les structures réelles $L \subset \mathbb{C}^{2}$ pour lesquelles $\langle$,$\rangle est réel sur L$ forment un espace homogène sous $\operatorname{SL}(2, \mathbb{C})$, avec $\operatorname{SL}(2, \mathbb{R})$ le stabilisateur de $\mathbb{R}^{2}$. Chacune définit une structure réelle $H_{L}:=L . U^{1}$ sur $H$, donc une conjugaison complexe $\sigma_{L}$ de points fixes $L U^{1}$.

Les disques dans $S$ forment de même un espace homogène sous $\operatorname{SL}(2, \mathbb{C})$, le stabilisateur de celui associé à $H_{\mathbb{R}}$ étant $\mathrm{SL}(2, \mathbb{R})$. La correspondance

$$
L \mapsto X(L):=\left\{D \mid\left(d, \sigma_{L}(d)\right)>1 \text { pour } d \text { un générateur de } D\right\}
$$


est donc une bijection de l'espace des structures réelles considérées avec l'espace des disques de $S$.

Le passage d'un disque au disque complémentaire se traduit sur les structures réelles en $L \mapsto i L$.

10. Les calculs du $n^{\circ} 3$ admettent l'interprétation suivante. Soit $X$ un disque (ouvert) de $S$, correspond à une structure réelle $L$, d'où des formes réelles $H_{L}$ de $H$ et $\operatorname{SL}(L)$ de $\operatorname{SL}(2, \mathbb{C})$. Alors $\Gamma\left(\bar{X}, \mathscr{W}^{0}\left(-\frac{1}{2}\right)^{\nabla=0}\right)$ admet une structure pré-hilbertienne $H_{L}$-invariante, le complété $\Gamma\left(\bar{X}, \mathscr{W}^{0}\left(-\frac{1}{2}\right)^{\nabla=0}\right)$ étant la représentation unitaire irréductible de $H_{L}$. On a

$$
\Gamma\left(\bar{X}, \mathscr{W}^{0}\left(-\frac{1}{2}\right)^{\nabla=0}\right) \subset \Gamma\left(\bar{X}, \mathscr{W}^{0}\left(-\frac{1}{2}\right)^{\nabla=0}\right) \subset \Gamma\left(X, \mathscr{W}^{0}\left(-\frac{1}{2}\right)^{\nabla=0}\right) .
$$

Le revêtement double $\operatorname{SL}(L)^{\sim}$ de $\operatorname{SL}(L)$ agit sur $\mathscr{O}\left(-\frac{1}{2}\right) \mid X$, de façon compatible à son action sur $\mathscr{O}(-1)$, d'où une action de $\operatorname{SL}(L)^{\sim} \operatorname{sur} \Gamma\left(\bar{X}, \mathscr{W}^{0}\left(-\frac{1}{2}\right)^{\nabla=0}\right)$. C'est celle qui normalise l'action de $H_{L}$, et induit l'action naturelle de $\operatorname{SL}(L)$ sur $H_{W}$.

La sous-représentation $\Gamma\left(\bar{X}, \mathscr{W}^{0}\left(-\frac{1}{2}\right)^{\nabla=0}\right)$ peut s'interpréter comme l'espace des vecteurs analytiques réels pour l'action de $\operatorname{SL}(L)^{\sim}$.

Cette description montre que la représentation projective de $\operatorname{SL}(L)$ se prolonge au monoïde des $g \in \operatorname{SL}(2, \mathbb{C})$ tels que $g X \supset X$.

11. Tout ce qui précède se généralise de $\operatorname{SL}(2, \mathbb{C})$ à $S p(2 n, \mathbb{C})$ : remplacer $\mathbb{C}^{2}$ par $\mathbb{C}^{2 n}$, muni d'une forme alternée non dégénérée $\langle$,$\rangle réelle sur \mathbb{R}^{2 n}, H$ par $\mathbb{C}^{2 n} \times \mathbb{C}^{*}$ avec la loi de groupe

$$
(x, \lambda)(y, \mu)=(x+y, \lambda \mu \exp (\pi i\langle x, y\rangle)),
$$

$S$ par l'espace des sous-espaces lagrangiens (= isotropes maximals) $D$ de $\mathbb{C}^{2 n}$. Le disque attaché à une structure réelle devient l'espace de Siegel des $D$ avec $|(d, \bar{d})|>1$ pour $d \neq 0$ dans $D$. Le faisceau inversible $\mathscr{O}\left(-\frac{1}{2}\right)$ est à remplacer $\operatorname{par} \operatorname{det}(D)^{1 / 2}$.

12. Remarque. A côté de représentations de $H$ où $\mathbb{C}^{*}$ agit par multiplication par $\lambda$, considérons de même des représentations où l'action est par $\lambda^{-1}$. On définit un fibré holomorphe $\mathscr{W}_{-}^{0}$ de telles représentations sur $S$, comme au $n^{\circ} 2$ et sur $\mathscr{W}_{-}^{0}\left(-\frac{1}{2}\right)$, on dispose à nouveau d'une connexion, donnée par une équation de la chaleur.

Sur un ouvert connexe et simplement connexe $U$ de $S$, considérons $W=$ $\mathscr{W}^{0}\left(-\frac{1}{2}\right)$, sa connexion et l'action de $H$. Dans $\Gamma(U, W)^{\nabla=0}$, on dispose pour chaque $x \in U$ d'une forme linéaire fixe par la droite correspondante, définie à un facteur près : $f \mapsto f(e)$ pour $f \in \mathscr{W}_{x}^{\circ}$. Pour chaque $x \notin U$, il existe un vecteur $v(x)$ fixe par $D_{x}$ (dans le modèle de Schrödinger et pour $x$ correspondant à $E=$ axe de $x$, c'est la constante 1 ). Il est unique à un facteur près. 
Fixons la structure réelle $H_{\mathbb{R}}$, d'où une structure réelle $S_{\mathbb{R}} \subset S$ (un cercle) et une décomposition de $S-S_{R}$ en deux disques $U_{1}, U_{2}$. Alors.

(a) Le complexe conjugué de $\Gamma\left(\bar{U}_{1}, W\right)^{\nabla=0}$ est $\Gamma\left(\bar{U}_{2}, W_{-}\right)^{\nabla=0}$.

(b) La forme hermitienne sur $\Gamma\left(\bar{U}_{1}, W\right)^{\nabla=0}$, invariante par $H_{\mathbb{R}}$, se réinterprète comme un accouplement bilinéaire entre $\Gamma\left(\bar{U}_{1}, W\right)^{\nabla=0}$ et $\Gamma\left(\bar{U}_{2}, W_{-}\right)^{\nabla=0}$, invariant par $H$.

Question. Soit $C$ une courbe de Jordan dans $S$, séparent $S-C$ en deux ouverts $U_{1}$ et $U_{2}$. Existe-t-il une unique forme bilinéaire séparante $H$-invariante accouplant $\Gamma\left(\bar{U}_{1}, W\right)^{\nabla=0}$ et $\Gamma\left(\bar{U}_{2}, W_{-}\right)^{\nabla=0}$, induite par une dualité parfaite entre $\Gamma\left(\bar{U}_{1}, W\right)^{\nabla=0}$ et $\Gamma\left(U_{2}, W_{-}\right)^{\nabla=0}$ ?

\section{BIBLIOGRAPHIE}

1. D. Shale, Linear symmetries of free Boson fields, Trans. Amer. Math. Soc. 103 (1962), 149-167.

2. J. von Neumann, Die Eindeutigkeit der Schrödingerschen operatoren, Ann. Mat. Pura Appl. (4) 104 (1931), 570-578.

3. A. Weil, Sur certains groupes d'opérateurs unitaires, Acta Math. 111 (1964), 143-211.

School of Mathematics, Institute for Advanced Study, Princeton, New Jersey 08540 